\title{
Avoiding state explosion by composition of minimal covering graph $\mathrm{s}_{1}$
}

\author{
Alain FINKEL \\ LIFAC \\ Ecole Normale Supérieure de Cachan \\ 61 Avenue du Président Wilson \\ F-94235 CACHAN cedex \\ Laure PETRUCCI \\ CEDRIC-IIE \\ 18 allée Jean Rostand, BP 77 \\ F-91002 EVRY cedex
}

\begin{abstract}
In this paper, we study composition and decomposition of Petri nets via a common set of places or transitions. We are interested in automatical verification of Petri nets properties from the point of view of reusability of partial results already obtained. We give two algorithms which allow to compute the minimal covering graph of a Petri net by composing the minimal covering graphs of each of its modules.
\end{abstract}

\section{Introduction}

Composition/Decomposition of Petri nets is a key problem, as well as concerns specification as verification of properties. This problem was already tackled in [Bou90], [Hac76], [Sou90], ..., but generally sub-classes of Petri nets were studied (state machine decomposable nets, bounded nets). When a system is specified with general Petri nets, one does not know, a priori, whether the net is bounded or not. Moreover, imposing to the places to be bounded may introduce unwanted deadlocks and change the demanded service.

We studied in [FP91] the properties preserved by composition/decomposition mechanisms. The techniques considered consist in composing two nets by fusion of common transitions or fusion of common places. They are applied to Petri nets in general, i.e. without any restricition. However some properties are not shared by a net and its subnets : two subnets can have the same property $P$, and their composed net can eventually not satisfy $P$. The main analysis methods for Petri nets are the calculus of invariants or of the covering graph. The first method does not allow to automatically deduce properties of the Petri net. But we know that the covering graph of a net allows to verify most properties. However, in some cases, the computation of the covering graph is too long. Our approach

\footnotetext{
1 Work supported by the Esprit Project DEMON (BRA 3148) and $C^{3}$.
} 
consists in using the minimal covering graph (which is the smallest covering graph) and to compute it not with the usual global and centralised method, but with a local and distributed method using a given decomposition of the Petri net.

So, in this paper, we study composition of minimal covering graphs of Petri nets composed via a common set of places or a common set of transitions. More precisely, we are interested in the construction of the minimal covering graph of a net using the minimal covering graphs of its subnets. Let us notice that a first study from Bourguet ([Bou90]) dealt with composition of reachability graphs for bounded Petri nets. In the case of unbounded Petri nets, covering graphs are needed. As the minimal covering graph of a net is both unique and as small as possible, composition of minimal covering graphs can easily be performed.

Basic notions about Petri nets can be found in [Rei85]. We first recall some notions about the minimal covering graph of a net. Then, we give two new algorithms which compute the minimal covering graph of a net from those of its subnets. The efficiency of these algorithms, as concerns time, can be increased by parallelising the calculus of the minimal covering graphs of each of the subnets.

\section{The minimal covering graph of a Petri net}

The classical Karp-Miller covering graph ([KM69]) is sometimes too large to be computed. The minimal covering graph ([Fin90]) is the smallest covering graph which allows to verify the same properties as the usual covering graph.

Definition 2.1 ([Fin90])

A coverability set $\mathrm{CS}(\mathrm{PN})$ of a Petri net $\mathrm{PN}=\left\langle\mathrm{P}, \mathrm{T}, \mathrm{V}, \mathrm{M}_{0}\right\rangle$ is a subset of $(\mathrm{N} \cup\{\omega\})^{\mathrm{P}}$ such that the two following conditions hold :

(i) for every reachable marking $m \in R S(P N)$, there is a marking $m^{\prime} \in C S(P N)$ such that $m \leq m^{\prime}$,

(ii) for every marking $m^{\prime} \in C S(P N)-R S(P N)$, there is an infinite strictly increasing sequence of reachable markings $\left\{m_{n}\right\}$ converging to $m^{\prime}$.

A coverability set $\mathrm{CS}(\mathrm{PN})$ is minimal iff no proper subset of $\mathrm{CS}(\mathrm{PN})$ is a coverability set of $\mathrm{PN}$.

\section{Lemma 2.2([Fin90])}

The minimal coverability set is finite and unique.

\section{Definition 2.3([Fin90])}

A coverability graph of a Petri net $\mathrm{PN}=\left\langle\mathrm{P}, \mathrm{T}, \mathrm{V}, \mathrm{M}_{0}\right\rangle$ is a labelled directed graph $\left.\angle \mathrm{N}, \mathrm{L}, \mathrm{A}\right\rangle$ where the set of nodes $N$ is a coverability set of $P N, L=T$ and there is an arc $\left(m, t, m^{\prime}\right) \in A$ iff $m\left(t>m^{\prime}\right.$. The minimal coverability graph of a Petri net PN is the coverability graph such that its set of nodes is the minimal coverability set. 
The algorithm to construct the minimal covering graph of a net can be found in [Fin90]. However, the algorithms we will present in the next sections are very similar, as they are derived versions.

\section{Theorem 2.4 ([Fin90])}

Let $P N$ be a Petri net and MCG(PN) its minimal covering graph.

(i) The reachability tree $\mathrm{RT}(\mathrm{PN})$ is infinite iff there is at least one circuit in $\mathrm{MCG}(\mathrm{PN})$.

(ii) The reachability set $\mathrm{RS}(\mathrm{PN})$ is infinite iff there is at least one symbol $\omega$ in $\mathrm{MCG}(\mathrm{PN})$.

(iii) A place $p$ is not bounded iff there is at least one marking $m \in M C G(P N)$ such that $m(p)=\omega$.

(iv) A transition $t$ is quasi-live iff there is at least one marking $m \in M C G(P N)$ such that for every place $p, m(p) \geq \operatorname{Pre}(p, t)$.

\section{Proposition 2.5 ([Fin90])}

The minimal covering graph is computable, finite and unique.

We are interested in composing nets either by fusion of common transitions or by fusion of common places. The definitions and properties of these compositions were introduced in [FP91].

We first recall the composition by fusion of transitions.

We consider two nets with distinct places and two kinds of transitions : proper ones, and transitions common to both nets. So, we study two nets $\mathrm{N}_{1}$ and $\mathrm{N}_{2}$ such that :
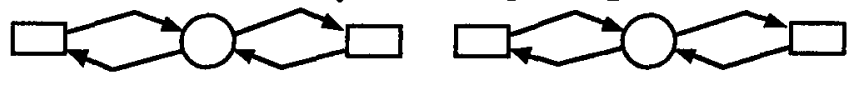

T1

P1

Tc Tc

P2

T2

where $T_{1}\left(T_{2}\right)$ is the set of transitions proper to $N_{1}\left(N_{2}\right), T_{c}$ is the set of transitions common to both nets, and $P_{1}\left(P_{2}\right)$ is the set of places of $N_{1}\left(N_{2}\right)$.

We obtain, by fusion of transitions common to $\mathrm{N}_{1}$ and $\mathrm{N}_{2}$, the following net $\mathrm{N}_{0}$ :

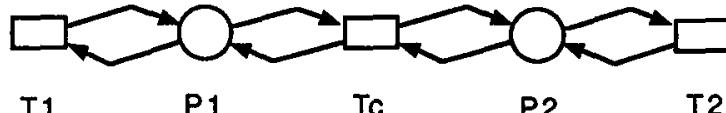

Composition by fusion of places is defined in a similar way. We consider two nets with distinct transitions and two kinds of places : proper ones, and places common to both nets. So, we study two nets $N_{1}$ and $N_{2}$ such that :

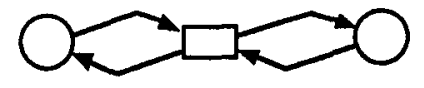

P1

T1

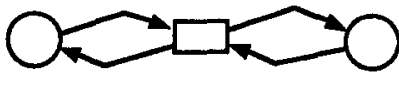

Pc
T2

P2

where $P_{1}\left(P_{2}\right)$ is the set of places proper to $N_{1}\left(N_{2}\right), P_{c}$ is the set of places common to both nets, and $\mathrm{P}_{1}\left(\mathrm{P}_{2}\right)$ is the set of transitions of $\mathrm{N}_{1}\left(\mathrm{~N}_{2}\right)$. 
We obtain, by fusion of places common to $\mathrm{N}_{1}$ and $\mathrm{N}_{2}$, the following net $\mathrm{N}_{0}$ :

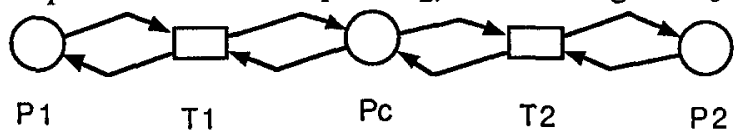

Notation : every marking $M$ of $N_{0}$ can be decomposed into $M=\left(M_{1}, M_{c}, M_{2}\right)$ where $M_{1}$ is the marking of places of $P_{1}, M_{2}$ is the marking of places of $P_{2}$ and $M_{c}$ is the marking of places of $P_{c}$.

\section{Fusion of transitions}

We now build the minimal covering graph of a net $\mathrm{N}_{0}$ obtained by composing two subnets $\mathrm{N}_{1}$ and $\mathrm{N}_{2}$ by fusion of common transitions. A marking $\mathrm{M}$ of net $\mathrm{N}_{0}$ can be divided into two parts : a marking $M_{1}$ corresponding to places of $P_{1}$ and a marking $M_{2}$ corresponding to places of $P_{2}$.

The construction of MCG0, minimal covering graph of net $\mathrm{N}_{0}$, from MCG1 and MCG2, minimal covering graphs of nets $\mathrm{N}_{1}$ and $\mathrm{N}_{2}$, is done using the following algorithm :

MCG1 := minimal covering tree of $\mathrm{N}_{1}$;

MCG2 := minimal covering tree of $\mathrm{N}_{2}$;

$/ *$ construction of MCG0 := minimal covering graph of $\mathrm{N}_{0} * /$

unprocessed_nodes $:=\left\{\right.$ create_node $\left.\left(r, M_{0}\right)\right\} ; \quad /^{*} \mathrm{M}_{0}=\left(\mathrm{M}_{1}, \mathrm{M}_{2}\right)$ is the marking of root $\mathrm{r} *$ /

processed_nodes : $=\varnothing$;

while unprocessed_nodes $\neq \emptyset$;

select some node $\mathrm{n} \in$ unprocessed_nodes;

unprocessed_nodes := unprocessed_nodes $-\{\mathrm{n}\} ; /^{*} \mathrm{~m}$ is the marking of $\mathrm{n}$ and $\mathrm{m}^{\prime}$ the one of $\mathrm{n}^{\prime} * /$ case $n:[1 . .4]$ of

1: there exists a node $n^{\prime} \in$ processed_nodes such that $m=m^{\prime}$ :

processed_nodes := processed_nodes $+\{\mathrm{n}\}$;

exit;

2: there exists a node $\mathrm{n}^{\prime} \in$ processed_nodes such that $\mathrm{m}<\mathrm{m}^{\prime}$ :

remove_node(n; MCT);

exit;

3: there exists a node $n^{\prime} \in$ processed_nodes such that $m>m^{\prime}$ :

$\mathrm{m}_{2}:=\mathrm{m}$;

ancestor $:=$ false;

for all ancestors $n_{1}$ of $n$ such that $m_{1}<m$ do

for all places $p$ such that $m 1(p)<m(p)$ do

$\prod_{\text {endfor }} \mathrm{m}_{2}(\mathrm{p}):=\omega$;

endfor

if there exists an ancestor $n_{1}$ of $n$ such that $m_{1}<m_{2}$

then ancestor := true;

$\mathrm{n}_{1}:=$ first processed node, on the path from the root to $\mathrm{n}$ such that $\mathrm{m}_{1}<\mathrm{m}_{2}$;

$\mathrm{m}_{1}:=\mathrm{m}_{2}$;

remove_tree $\left(\mathrm{n}_{1} ; \mathrm{MCT}\right)$;

remove from (processednodes + unprocessednodes) all nodes of tree $\left(\mathrm{n}_{1} ; \mathrm{MCT}\right)$;

unprocessednodes $:=$ unprocessednodes $+\left\{n_{1}\right\}$;

endif

for every $\mathrm{n}_{1} \in$ processednodes such that $\mathrm{m}_{1}<\mathrm{m}_{2}$ do 


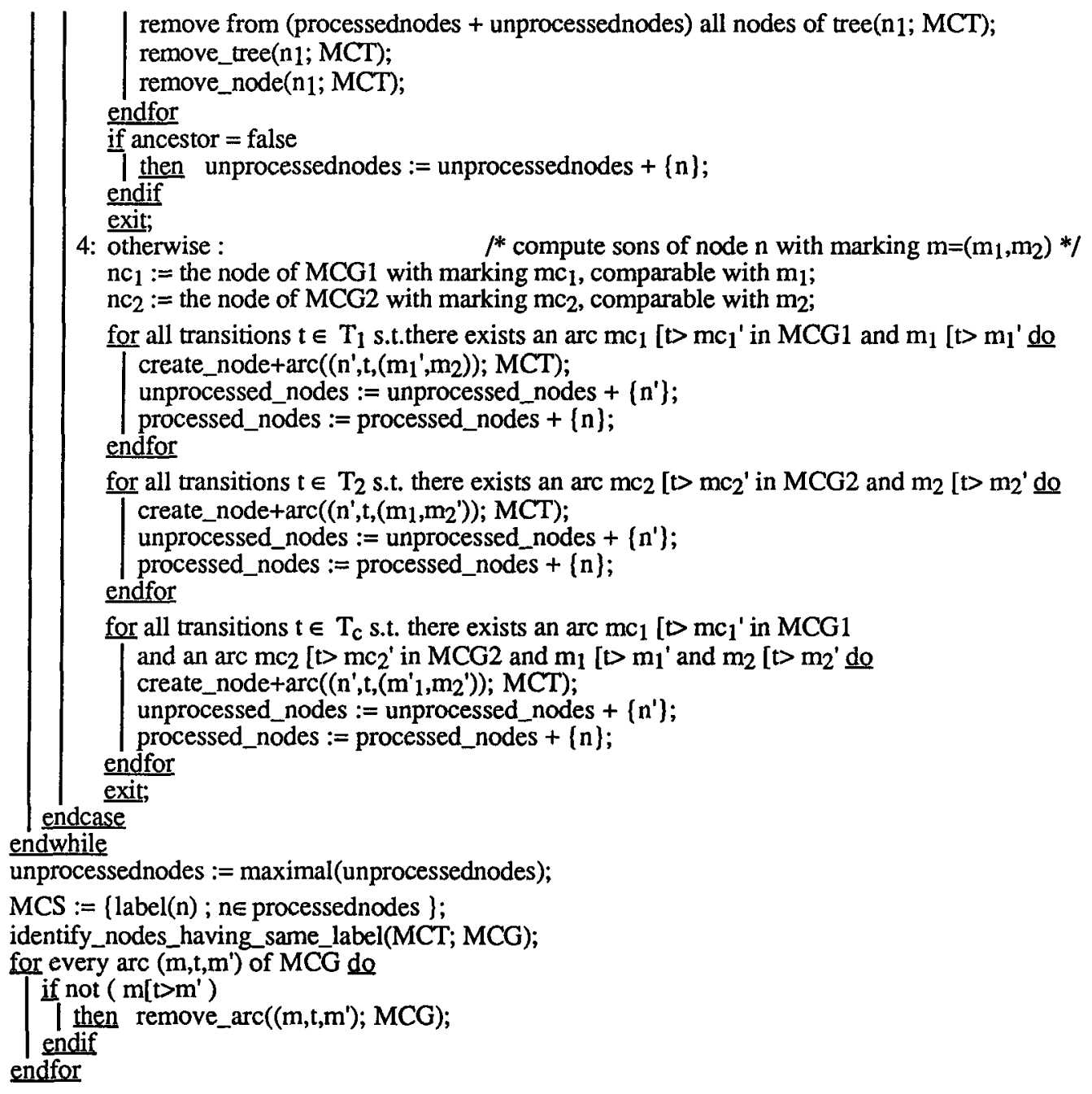

\section{Informal explanation :}

We start with the initial marking $M_{0}=\left(M_{01}, M_{02}\right)$ of $N_{0}$. There exists one and only one marking $M_{1}$ comparable with $\mathrm{M}_{0_{1}}$ (related by the < relation) in MCG1 (property of the minimal covering graph). Likewise, there exists a unique marking $\mathrm{M}_{2}$ comparable with $\mathrm{M}_{2}$ in $\mathrm{MCG}$. If there exists an arc $M_{1}\left[t>\ldots\right.$ in $M C G 1$, with $t \in T_{1}$, then $t$ may be firable in $N_{0}$ and, if it is the case, a similar arc is constructed in MCG0. If there exists an $\operatorname{arc} \mathrm{M}_{2}\left[\mathrm{t}>\ldots\right.$ in MCG2, with $t \in \mathrm{T}_{2}$, then $\mathrm{t}$ may be firable in $\mathrm{N}_{0}$ and, if it is the case, a similar arc is constructed in MCG0. If there exists an arc $\mathrm{M}_{1}[\mathrm{t}>\ldots$ in MCG1 and an $\operatorname{arc} M_{2}\left[t>\ldots\right.$ in $M C G 2$, with $t \in T_{c}$, then $t$ may be firable in $N_{0}$, and if it the case, the arc is constructed.

This is not only valid for the initial marking, but also for any reachable marking of $\mathrm{N}_{0}$. To build MCG0, we already know which transitions are firable, and also part of the marking - the one 
corresponding tothe set of places not concerned by the firing of the transition, i.e. places of $\mathrm{P}_{2}$ for a transition of $T_{1}$ and places of $P_{1}$ for a transition of $T_{2}$.

\section{Proposition 3.1}

The algorithm terminates.

\section{Fusion of places}

As for composition by fusion of transitions, we are going to study composition of minimal covering graphs when using composition of nets by fusion of common places.

A marking $\mathrm{M}$ of $\mathrm{N}_{0}$ can be decomposed into three parts : a marking $\mathrm{M}_{1}$ corresponding to places of $P_{1}$, a marking $M_{c}$ corresponding to places of $P_{c}$ and a marking $M_{2}$ corresponding to places of $P_{2}$.

We propose the following algorithm to compute the minimal covering graph of the entire net from those of the subnets:

finished := false;

$\forall \mathrm{i}, \mathrm{k}_{\mathrm{c}_{\mathrm{i}}}:=\mathrm{M}_{0}\left(\mathrm{p}_{\mathrm{c}_{\mathrm{i}}}\right)$;

$/ *$ initial number of tokens in common place $\mathrm{p}_{\mathrm{i}}{ }^{*} /$

while not finished do

$\mathrm{MCG1}=$ minimal covering tree of $\mathrm{N}_{1}$, with $\mathrm{M}_{01}\left(\mathrm{p}_{\mathrm{c}_{\mathrm{i}}}\right)=\mathrm{k}_{\mathrm{C}_{\mathrm{j}}}$;

$\mathrm{MCG} 2=$ minimal covering tree of $\mathrm{N}_{2}$, with $\mathrm{M}_{2}\left(\mathrm{p}_{\mathrm{c}_{\mathrm{i}}}\right)=\mathrm{k}_{\mathrm{c}_{\mathrm{i}}}$;

$\forall \mathrm{i}, \mathrm{k}_{\mathrm{C}_{11}}:=\max \mathrm{MCGl} M\left(\mathrm{p}_{\mathrm{c}_{\mathrm{i}}}\right) ; \quad \quad / *$ the higher number of tokens in $\mathrm{p}_{\mathrm{ci}}$ in MCG1 $* /$

$\forall \mathrm{i}, \mathrm{k}_{\mathrm{c}_{2}}:=\max \mathrm{MCG} 2 \mathrm{M}\left(\mathrm{p}_{\mathrm{c}_{1}}\right) ; \quad \quad{ }^{*}$ the higher number of tokens in $\mathrm{p}_{\mathrm{ci}}$ in $\mathrm{MCG} 2 * /$

if $\forall \mathrm{i}, \mathrm{k}_{\mathrm{C}_{11}}=\mathrm{k}_{\mathrm{C}_{\mathrm{i}}}$

$\mid$ then finished := true; $\quad / *$ the bounds of places $\mathrm{p}_{\mathrm{i}}$ are the same in both nets $* /$

endif

$\forall \mathrm{i}, \mathrm{k}_{\mathrm{ci}_{1}}:=\max \left(\mathrm{k}_{\mathrm{c}_{\mathrm{i} 1}}, \mathrm{k}_{\mathrm{c}_{\mathrm{i} 2}}\right)$;

if there exists a sequence $s$ and $i, j, i \neq j$, s.t.:

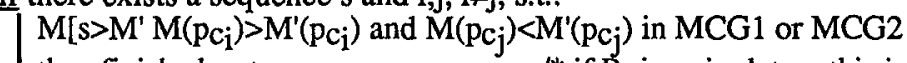

then finished := true; $\quad f^{*}$ if $\mathrm{P}_{\mathrm{C}}$ is a singleton, this is not applied $* /$

endif

endwhile

/* construction of $\mathrm{MCG0}:=$ minimal covering graph of $\mathrm{N}_{0} * /$

unprocessed_nodes := (create_node $\left.\left(r, \mathrm{M}_{0}\right)\right\} ; \quad I^{*} \mathrm{M}_{0}=\left(\mathrm{M}_{1}, \mathrm{M}_{2}\right)$ is the marking of root $\mathrm{r} * /$ processed_nodes : $=\varnothing$;

while unprocessed_nodes $\neq \varnothing$;

select some node $\mathrm{n} \in$ unprocessed_nodes;

unprocessed_nodes $:=$ unprocessed_nodes $-\{n\} ; / * m$ is the marking of $n$ and $m^{\prime}$ the one of $n^{\prime} * /$ case $\mathrm{n}:[1 . .4]$ of

1: there exists a node $n^{\prime} \in$ processed_nodes such that $m=m^{\prime}$ :

processed_nodes := processed_nodes $+\{n\}$;

exit;

2: there exists a node $n^{\prime} \in$ processed_nodes such that $m<\mathrm{m}^{\prime}$ : remove_node(n; MCT);

exit; 
3: there exists a node $n^{\prime} \in$ processed_nodes such that $m>m^{\prime}$ :

$\mathrm{m}_{2}:=\mathrm{m}$;

ancestor $:=$ false;

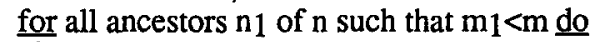

for all places $\mathrm{p}$ such that $\mathrm{m} 1(\mathrm{p})<\mathrm{m}(\mathrm{p})$ do

$\prod_{\text {endfor }} \mathrm{m}_{2}(\mathrm{p}):=\omega$

endfor

if there exists an ancestor $n_{1}$ of $n$ such that $m_{1}<m_{2}$

then ancestor := true;

$\mathrm{n}_{1}:=$ first processed node, on the path from the root to $\mathrm{n}$ such that $\mathrm{m}_{1}<\mathrm{m}_{2}$;

$\mathrm{m}_{1}:=\mathrm{m}_{2}$

remove_tree(n1; MCT);

remove from (processednodes + unprocessednodes) all nodes of tree( $\left.\mathrm{n}_{1} ; \mathrm{MCT}\right)$;

unprocessednodes $:=$ unprocessednodes $+\left\{\mathrm{n}_{1}\right\}$;

endif

for every $n_{1} \in$ processednodes such that $m_{1}<m_{2}$ do

remove from (processednodes + unprocessednodes) all nodes of tree(n1; MCT);

remove_tree(n1; MCT);

remove_node(n1; MCT);

endfor

if ancestor $=$ false

then unprocessednodes $:=$ unprocessednodes $+\{n\}$;

endif

exit;

4: otherwise :

* compute sons of node $n$ with marking $m=\left(m_{1}, m_{c}, m_{2}\right) * /$

$\mathrm{nc}_{1}:=$ node in one of the MCGl computed, of marking comparable with $\left(\mathrm{m}_{1}, \mathrm{~m}_{\mathrm{c}}\right)$;

$\mathrm{nc}_{2}:=$ node in one of the MCG2 computed, of marking comparable with $\left(\mathrm{m}_{2}, \mathrm{~m}_{\mathrm{c}}\right.$ );

if $\mathrm{nc}_{1}=0$

$f^{*}$ in both cases, value 0 is retumed if the node could not be found */

then for all $t \in T_{1}$ such that $\left(m_{1}, m_{c}\right)\left[t>\left(m_{1}{ }^{\prime}, m_{c}{ }^{\prime}\right)\right.$ do

create_node+arc $\left(\left(n^{\prime}, t,\left(m_{1}^{\prime}, m_{c^{\prime}}, m_{2}\right)\right) ; M C T\right)$;

unprocessed_nodes $:=$ unprocessed_nodes $+\left\{n^{\prime}\right\}$;

processed_nodes $:=$ processed_nodes $+[\mathrm{n}]$;

endfor

else for all $t \in T_{1}$ s.t. there exists an $\operatorname{arc}\left(\mathrm{mc}_{1}, \mathrm{mc}_{\mathrm{c}}\right)\left[\nabla\left(\mathrm{mc}_{1}{ }^{\prime}, \mathrm{mc}_{\mathrm{c}}{ }^{\prime}\right)\right.$

in one of the MCG1 computed and $\left(m_{1}, m_{c}\right)\left[t>\left(m_{1}^{\prime}, m_{c}^{\prime}\right)\right.$ do

create_node+arc $\left(\left(n^{\prime}, t,\left(m_{1}^{\prime}, m_{c}^{\prime}, m_{2}\right)\right) ; M C T\right)$;

unprocessed_nodes $:=$ unprocessed_nodes $+\left\{n^{\prime}\right\}$;

processed_nodes $:=$ processed_nodes $+[n\}$

endif

endfor

if $n c_{2}=0 \quad l^{*}$ both "if" are similar, but they are duplicated for easier reading */

then for all $t \in T_{2}$ such that $\left(m_{2}, m_{c}\right)\left[t>\left(m_{2}^{\prime}, m_{c}\right)\right.$ de

create_node+arc $\left(\left(n^{\prime}, t,\left(m_{1}, m_{c}{ }^{\prime}, m_{2}{ }^{\prime}\right)\right) ; M C T\right)$;

unprocessed_nodes $:=$ unprocessed_nodes $+\left\{n^{\prime}\right\}$;

processed_nodes := processed_nodes $+[\mathrm{n}\}$;

endfor

else for all $t \in T_{2}$ such that there exists an $\operatorname{arc}\left(\mathrm{mc}_{2}, \mathrm{mc}_{\mathrm{c}}\right)\left[t>\left(m c_{2}{ }^{\prime}, \mathrm{mc}_{\mathrm{c}}{ }^{\prime}\right)\right.$

in one of the MCG2 computed and $\left(m_{2}, m_{c}\right)\left[t>\left(m_{2}^{\prime}, m_{c}{ }^{\prime}\right)\right.$ do

create_node $+\operatorname{arc}\left(\left(n^{\prime}, t,\left(m_{1}, m_{c}^{\prime}, m_{2}^{\prime}\right)\right) ; M C T\right) ;$

unprocessed_nodes $:=$ unprocessed_nodes $+\left[n^{\prime}\right\}$;

processed_nodes $:=$ processed_nodes $+\{\mathrm{n}\}$; 


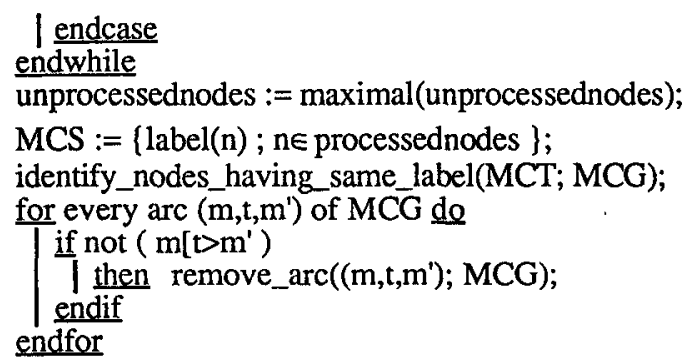

Informal explanation:

In the first loop, we compute the minimal covering graphs of each module. One of the subnets can add tokens to a common place. In this case the number of tokens in this place will be greater then the initial marking. So, these tokens can be used by the other subnet. Thus, we have to take this information into account.

The union of minimal covering graphs is defined as follows. We start with the initial state $\mathrm{M}_{0}$ of net $N_{0}$. It can be decomposed into three parts : $M_{0}=\left(M_{1}, M_{c}, M_{2}\right)$, where $M_{1}$ (resp. $M_{2}$ ) is the marking of places of $P_{1}$ (resp. $P_{2}$ ) and $M_{c}$ is the marking of places of $P_{c}$, common to both nets. Let us suppose that in one of the MCG1 (resp. MCG2) computed, there exists a marking $\left(M_{1}{ }^{\prime}, M_{c}{ }^{\prime}\right.$ ) (resp. $\left(M_{2}{ }^{\prime}, M_{c}{ }^{\prime}\right)$ ) comparable with $\left(M_{1}, M_{c}\right)$ (resp. with $\left(M_{2}, M_{c}\right)$ ). Let $t$ be a transition of $T_{1}$ such that $\left(M_{1}{ }^{\prime}, M_{c}{ }^{\prime}\right)[t>$ in MCG1 (for MCG2, the operation is similar). If $t$ is firable in the global net, then, we will obtain in $\mathrm{MCG} 0$ an $\operatorname{arc}\left(\mathrm{M}_{1}, \mathrm{M}_{\mathrm{c}}, \mathrm{M}_{2}\right)\left[\mathrm{t}>\left(\mathrm{M}_{1}{ }, \mathrm{M}_{\mathrm{c}}{ }^{\prime}, \mathrm{M}_{2}\right)\right.$. We apply the same operation for the markings obtained.

When processing a marking $M$, if a marking $M^{\prime}$ comparable with marking $M$, was found in one of the MCG1 computed, then only transitions of $T_{1}$ firable from $M$ are transitions firable from $M^{\prime}$. It is the same for transitions of MCG2.

\section{Proposition 5.1}

The algorithm terminates.

\section{Proof}

The problem of the algorithm termination is not as simple as for the algorithm concerning composition by fusion of transitions. So we are going to detail the proof. For that purpose, proving that the first loop of the algorithm terminates is enough.

Let $s$ be a generic term used to denote a firing sequence such that $M[s>M$ '. Two cases may be encountered:

- $\left(\forall \mathrm{s} \in \mathrm{T}_{\mathrm{i}}^{*}\right)\left(\left(\forall \mathrm{p} \in \mathrm{P}_{\mathrm{c}} \mathrm{M}^{\prime}(\mathrm{p}) \leq \mathrm{M}(\mathrm{p})\right)\right.$ or $\left.\left(\forall \mathrm{p} \in \mathrm{P}_{\mathrm{c}} \mathrm{M}(\mathrm{p})^{\prime} \geq \mathrm{M}(\mathrm{p})\right)\right)$.

Let us consider the first subcase : $\forall p \in P_{c} M^{\prime}(p) \leq M(p)$. The bound of places is preserved. So, the algorithm terminates.

Let us now consider the second subcase : $\forall p \in P_{c} M^{\prime}(p) \geq M(p)$. The nodes are uncomparable, by definition of the minimal covering graph. So, there exists a place $p^{\prime}$, not in $P_{c}$, such that $M\left(p^{\prime}\right)>M^{\prime}\left(p^{\prime}\right)$, i.e. the marking of $p^{\prime}$ decreases when firing s. So, the algorithm stops. 
- $\exists s \in T_{i}^{*}$ tq $\left(\left(\exists p \in P_{c}\right.\right.$ tq $\left.M^{\prime}(p)>M(p)\right)$ and $\left(\exists p^{\prime} \in P_{c}\right.$ tq $\left.\left.M^{\prime}\left(p^{\prime}\right)<M\left(p^{\prime}\right)\right)\right)$ : in this case, we force the algorithm termination by setting variable finished to true.

\section{Example}

Let us consider a railway with a two-way section. Trains circulate from East to West and from West to East. The driving rules on the two-way part of the railway are the following :

- there cannot be two trains going in opposite ways (otherwise, there would be collisions);

- there can be one or several trains going in the same direction;

- there are at most two trains on the section (for security reasons).

We model this problem with the following Petri net.

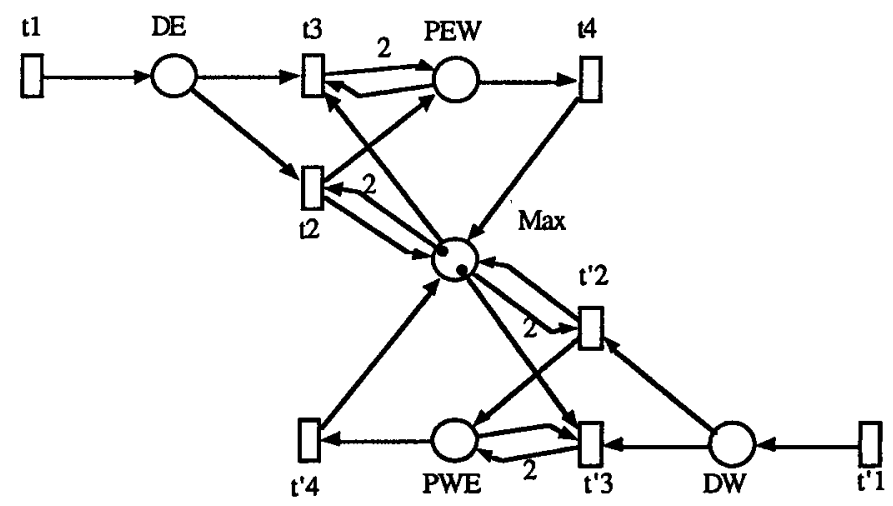

Trains going from East to West (from West to East) are generated by transition $t 1$ ( $t$ ' 1 ) and are then in their departure place, $D E(D W)$. The first train to go on the critical section fires transition $t 2\left(t^{\prime} 2\right)$, while the following one would fire transition $t 3\left(t^{\prime} 3\right)$. Place PEW (PWE) contains the trains going from East to West (from West to East) on the two-way part of the railway. Place Max contains the maximal number of trains which can still be allowed on this part. When a train wants to exit this system, it fires transition $t 4\left(t^{\prime} 4\right)$.

We are going to calculate the minimal covering graph of this net. We use, in order to compare the different methods, two time criteria : the tests of transitions (is a transition firable?) and the calculus of the marking of a place (what is the new marking of a place $p$ ?).

First, we do the direct construction of the graph. The cost of this operation is $56 \mathrm{tt}$ (tests of transitions) and $76 \mathrm{pm}$ (calculus of places markings).

Secondly, we decompose the net into two nets communicating via place Max : the first net NP1 is composed of places DE, PEW, Max and transitions $t 1, t 2, t 3, t 4$; the second net NP2 is composed of places DW, PWE, Max and transitions $t^{\prime} 1, t^{\prime} 2, t^{\prime} 3, t^{\prime} 4$. The cost of the whole operation is 38 t $t$ and $37 \mathrm{mp}$, when the covering graphs of the components are computed sequentially. When computed in parallel, the cost becomes $22 \mathrm{tt}$ and $20 \mathrm{mp}$. 
Thirdly, we decompose the net into three nets communicating via transitions : the first net NT1 is composed of place DE and transitions $\mathrm{t} 1, \mathrm{t} 2, \mathrm{t} 3$; the second net NT2 is composed of places PEW, PWE, Max and transitions $t 2, t 3, t^{\prime} 2, t^{\prime} 3$; the third net NT3 is composed of place DW and transitions $t^{\prime} 1, t^{\prime} 2, t^{\prime} 3$. The cost of the whole operation is $50 \mathrm{tt}$ and $33 \mathrm{mp}$, when the covering graphs of the components are computed sequentially. When computed in parallel, the cost is $38 \mathrm{tt}$ and $31 \mathrm{mp}$.

\section{Conclusion}

In this paper, we studied the composition of Petri nets by fusion of a set of common transitions or a set of common places. The goal of this work was to make verification of properties easier by reusing already computed results.

We presented two new algorithms to compute the minimal covering graph of a Petri net, knowing the minimal covering graphs of its modules.

The complexity (in terms of tests of firability for transitions) mainly depends on the chosen decomposition. If the decomposition of the net is badly chosen, the calculus by composition can be worse than a direct computation. The problem is thus to find out an optimal decomposition for a more efficient covering graph computation. In general, it would certainly be possible to obtain a decomposition such that the parallel computation of the $\mathbf{n}$ modules covering graphs would have almost the complexity of the direct computation divided by $n$. This problem was examplified in the previous section.

From a practical point of view, these algorithms will be implemented within the Petri nets environment PAPETRI ([BJP90]).

\section{Bibliography}

[BJP90] G. Berthelot, C. Johnen, L. Petrucci : PAPETRI : Environment for the Analysis of PETRI nets. Proceedings of the $2^{\text {nd }}$ Computer-Aided Verification Workshop, NewBrunswick, USA, 18-21 June 1990.

[Bou90] A. Bourguet : Etude de la concordance de comportement de deux réseaux de Petri. Application d la validation des protocoles : détection automatique des erreurs de conception. Thesis of University Pierre et Marie Curie (Paris 6), September 1990.

[Bra83] G.W. Brams : Réseaux de Petri : théorie et pratique. Masson Ed, 1983.

[Fin90] A. Finkel : The minimal coverability graph for Petri nets. Proceedings of the $11^{\text {th }}$ International Conference on Application and Theory of Petri nets, Paris, June 1990.

[FP91] A. Finkel, L. Petrucci : Verification of net properties by composition/decomposition. Research report CEDRIC, January 1991.

[Hac76] M. Hack : Decidability questions for Petri nets. PhD Thesis, Technical Report 161, MIT, Laboratory for Computer Science, Juin 1976.

[KM69] R.M. Karp, R.E. Miller : Parallel program schemata. JTSS 4, 1969, pp 147-195.

[Rei85] W. Reisig: Petri nets. Springer-Verlag, 1985.

[Sou90] Y. Souissi : Une étude de la préservation de propriétés par composition de réseaux de Petri. Quelques extensions aux réseaux d files. Application a la validation de protocoles de communication. Thesis of University Pierre et Marie Curie (Paris 6), February 1990. 
Minimal covering graph of net $\boldsymbol{N}$ :

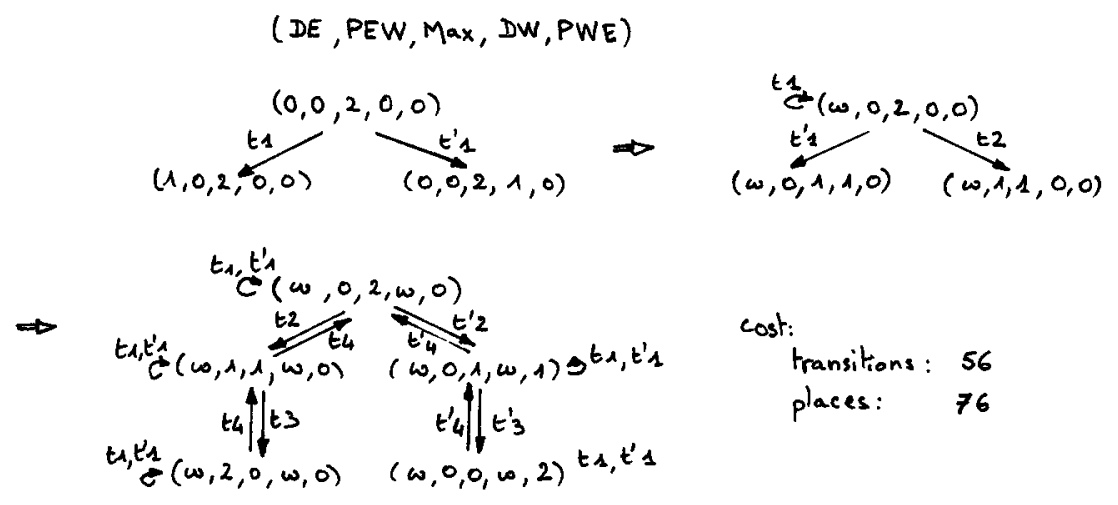

Minimal covering graph of net NP1: (NP2 with $t$; iustead of $t$ and maskings (DW, Pwe, Mex))

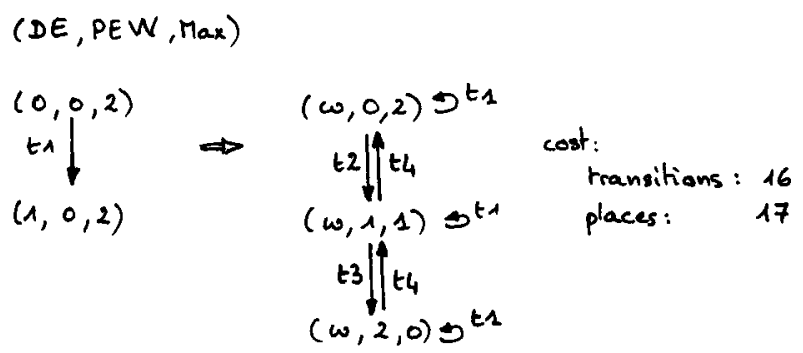

Composition of minimal covering graphs of NP1 and NP2:

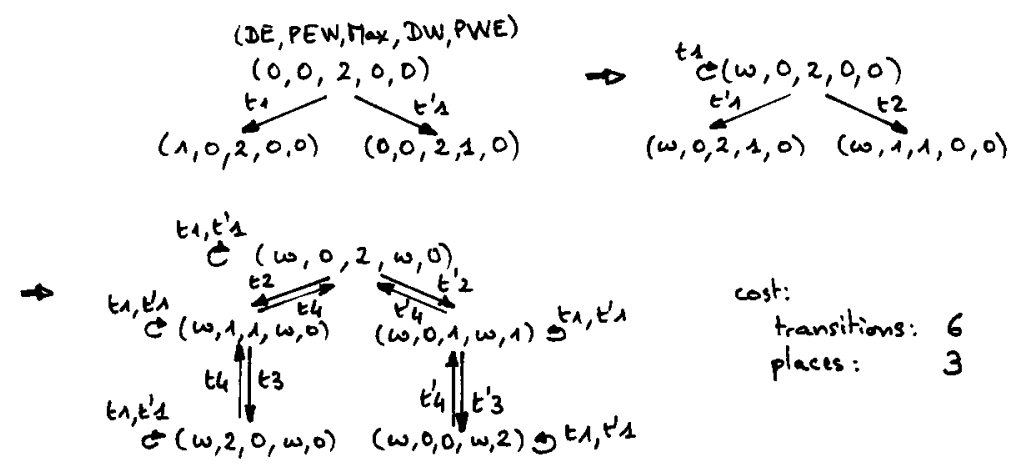


Minimal covering graph of net NT1: (NT3 with $t^{\prime}$ instead of $t$ and markings (DW))

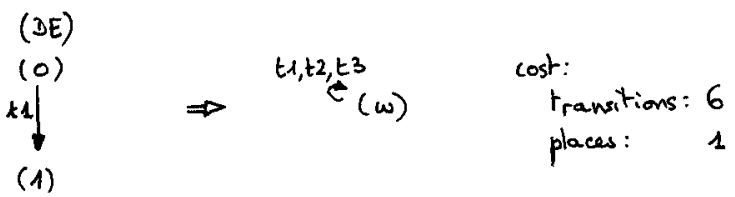

Minimal covering graph of net NT2:

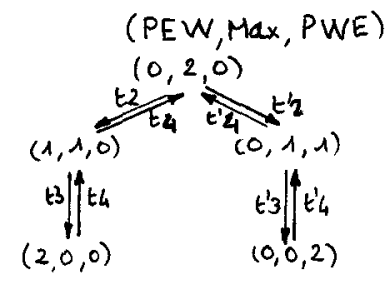

cost:

transitions: 30

places: $\quad 24$

Composition of minimal covering graphs of $N T 1$ and NT2.

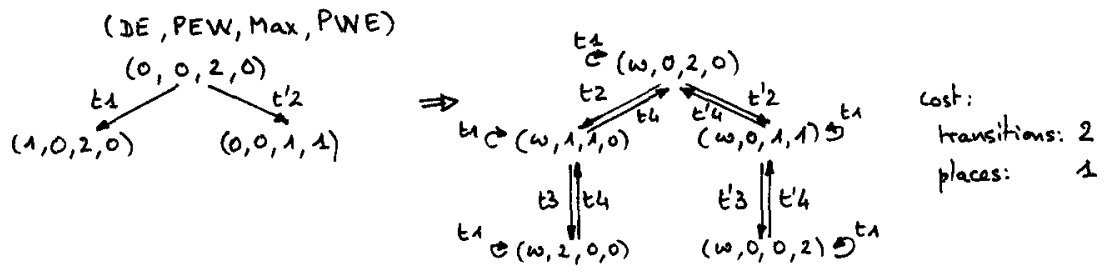

Composition with the minimal covering graph of NT3:

$$
\begin{aligned}
& \text { (DE, PEW, Max, DW, PWE) } \\
& (0,0,2,0,0) \\
& { }^{t A} C(\omega, 0,2,0,0) \\
& \underbrace{t 1}_{(1,0,2,0,0)} \underbrace{t_{1}^{\prime}}_{(0,0,2,1,0)} \Rightarrow \underbrace{t_{1}}_{(\omega, 0,2,1,0)} \underbrace{t_{2}}_{(\omega, 1,1,0,0)} \\
& E_{1, t_{1}}(\omega, 0,2, \omega, 0)
\end{aligned}
$$

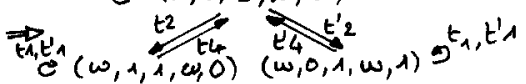

$$
\begin{aligned}
& t_{1,} t_{1} \quad t_{(\omega, 2,0, \omega, 0)} \|_{(\omega, 0,0, \omega, 2)} m^{t_{1}, t_{1} A} \\
& \text { cost: } \\
& \text { transitions: } 6 \\
& \text { places: } 6
\end{aligned}
$$

